\title{
Evaluation on the combined effect of Sesamin and Schisandra extract on blood fluidity
}

\author{
Daniel Tsi ${ }^{1, *}$ and Amabel Tan ${ }^{2}$ \\ ${ }^{1}$ BRAND'S Centre for Health and Nutritional Sciences, Cerebos Pacific Limited, 18 Cross Street \# 12-01/08, Singapore 048423; ${ }^{2}$ Department of \\ Microbiology and Immunology, The University of Melbourne,Victoria 3010, Australia; Daniel Tsi* - E-mail: danieltsi@yahoo.co.uk; \\ * Corresponding author
}

received December 21, 2007; accepted January 02, 2008; published January 27, 2008

\begin{abstract}
:
Several studies have demonstrated a link between blood viscosity and various forms of liver dysfunction. Therefore, we investigated the effect of liver protective herbal materials, Sesamin combined with extract of Schisandra chinensis berry (Schisandra) for its potential to improve blood fluidity in humans. Ten human subjects were recruited to study the effect of sesamin combined with schisandra extract ( $\mathrm{SCH}$ ) for two weeks on blood viscosity. Blood fluidity was measured as the transit time for $100 \mu \mathrm{l}$ of heparinized whole blood to pass through a micro-channel array setup at baseline, 1 week and 2 weeks. For safety assessment, blood biochemistry, hematology and urine analysis were taken at baseline, 1 week and 2 weeks after SCH administration. No safety concern and adverse effects were observed during the 2-week continuous intake period. Intake of SCH reduced blood passage time by $9.0 \%$ and $9.7 \%$ at 1 and 2 weeks, respectively. In conclusion, this pilot clinical study indicates that the combined administration of sesamin with schisandra extract could improve blood fluidity after 1 week of oral intake and this effect was sustained up to 2 weeks.
\end{abstract}

Keywords: Schisandra chinensis; sesamin; blood fluidity; blood passage time; micro-channel array

Background:

There is a common belief in the Southeast Asian Chinese community that a smooth blood circulation is important for good health. There are several "blood cleansing" Traditional Chinese Medicinal (TCM) herbs shown to improve blood circulation by reducing blood viscosity and promoting blood circulation in animal studies [1-2]. Blood viscosity or fluidity is influenced by circulating plasma lipoproteins [34], platelet activity and other physiological parameters [5]. Modulating these components, possibly by improving metabolic function might improve fluidity and blood flow. Several studies have demonstrated a link between these parameters, blood viscosity and various forms of liver dysfunction [6-8]. In linking traditional health concepts with scientific rationale, we hypothesize that liver protective 'blood cleansing' TCM herb materials may help to reduce blood viscosity.

Sesamin, a lignan that occurs exclusively in sesame seeds, has been reported to inhibit cholesterol absorption and synthesis [9], in addition to improving hepatic detoxification in rats [10]. Schisandra chinensis with common names like Schisandra, Magnolia vine and $\mathrm{Wu}$-wei-zi, similarly, has been reported to confer liver protective effect in a rat model of hepatotoxicity $[\mathbf{1 1}, \mathbf{1 2}]$ and is an established Chinese herbal medicine associated with liver protection and function [13]. In the present study, we examined the action of sesamin-schisandra extract combination for its potential to improve blood fluidity in humans.

\begin{abstract}
Methodology:
Subjects

Healthy subjects who had given written informed consent in accordance with the "Declaration of Helsinki" were screened for eligibility. Subjects eligible for the study showed "above average" blood passage time of $\geq 44.0 \mathrm{sec}$ when tested during screening at one week and two weeks prior to Day 0 . Subjects who failed to meet the criteria were excluded from the study. The 'normal' blood passage time of $\leq 44 \mathrm{sec}$ is derived from the mean value of 51 healthy male volunteers in a previous investigation (data not included).
\end{abstract}

Exclusion criteria included drug allergy, gastrointestinal disorder or surgery, respiratory or cardiac disorder, excessive intake of tobacco ( $>50$ cigarettes per day), alcohol (>equivalent of $120 \mathrm{ml}$ ethyl alcohol), significant irregular dietary habits, blood donation within two weeks of study initiation and any condition deemed unsuitable for study participation. Subjects would be withdrawn from the study in the event they need to take medication (such as aspirin, anticogulants) that might affect blood viscosity. Of the 20 subjects initially screened for each group, only ten subjects were found to be eligible.

\section{Study Design}

Ten healthy male subjects with mean age of 51 years (range 37 to 69), mean body weight of $75.8 \mathrm{~kg}$ (range 60.5 to 107.6) and mean BMI of 26.5 (range 21.0 to 37.6), participated in the study. In the 2-week, open labelled intervention trial with subjects acted as their own controls, each volunteer consumed 2 tablets of sesamin-schisandra combination ( $\mathrm{SCH})$ (Cerebos Pacific Limited, Singapore), 
twice daily. Each SCH tablet contains $2.5 \mathrm{mg}$ sesamin, 65 mg schisandra extract and 3.75 IU Vitamin E. The subjects were advised to maintain their regular dietary habits and physical activities, and avoid any supplement (such as ginkgo leaf extract) or medication (such as aspirin, anticogulants) that might affect blood viscosity.

The effect of SCH on blood fluidity was assessed at baseline (week 0), 1-week and 2-week after continuous oral intake. At each time point, fasting blood samples for blood fluidity assessment were taken in the morning before breakfast. Blood chemistry, hematology and urine tests for safety assessments were performed at screening ( 2 weeks prior to study) and 2 weeks post dosing.

\section{Measurement of Blood Viscosity or Fluidity}

Blood fluidity was assessed using a microchannel array flow analyser (MC-FAN) (Hitachi-Haramachi Electronics Co. Ltd) as described by Kikuchi (1995) [14]. Blood (5 ml) obtained by venpuncture of an antecubital vein was collected in vacuum sampler tubes added with $0.25 \mathrm{ml}$ heparin solution (1000 units $/ \mathrm{ml}$ ). The transit time for $100 \mu \mathrm{l}$ of whole blood to flow through the 8736-parallel-row micro-channel array (channel width $7 \mu \mathrm{m}$, length $30 \mu \mathrm{m}$, depth $4.5 \mu \mathrm{m}$; Bloody
6-7, Hitachi Haramachi Electronics, Japan) was measured at a pressure of $20 \mathrm{~cm} \mathrm{H}_{2} \mathrm{O}$. The microchannel flow analyser was calibrated with the saline before each measurement of blood passage time. The transit time was then converted into blood passage time to adjust for the transit time of saline. The blood passage time is defined as the ratio of "transit time for blood (sample) x $12 \mathrm{~s}$ " to "transit time for saline".

\section{Safety Assessment}

Haematology, blood biochemistry [total protein, albumin, total bilirubin, alanine aminotransferase (ALT), aspartate aminotransferase (AST), gamma-glutamyltransferase $(\gamma-\mathrm{GT})$, Alkaline phosphatase (ALP), Lactic dehydrogenase (LDH)], total cholesterol, HDL-cholsterol, LDL-cholesterol, triglycerides, fasting blood sugar and urine tests were performed 2 weeks before and after $\mathrm{SCH}$ administration.

\section{Statistical Analysis}

Distribution-free non-parametric statistical tests were employed. The Mann Whitney U test was used to compare the differences at different time points over 2 weeks. Blood transit time is expressed as Mean \pm Standard Error of Mean (SEM).

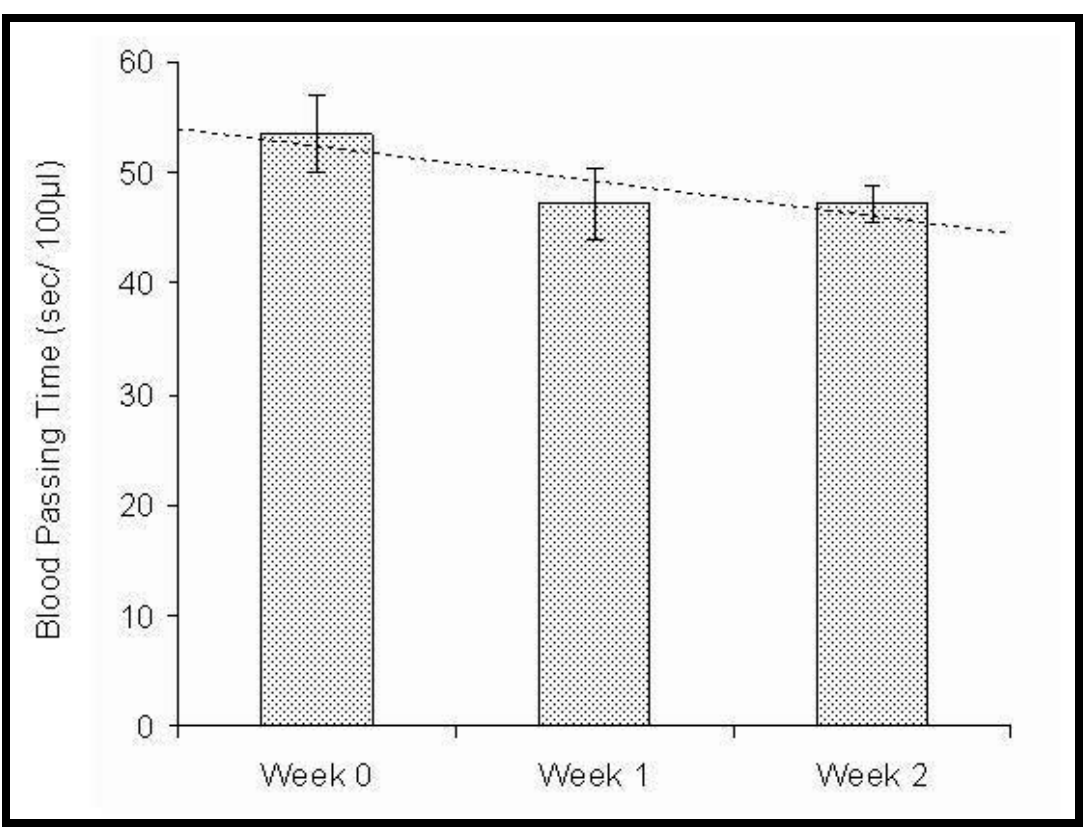

Figure 1: Effect of continuous intake of Schisandra for 2 weeks on the fasting blood passage time. There was a sustained decreasing trend in blood passage time during 2 weeks of Schisandra administration. Values represent Mean \pm SEM

\section{Results:}

Among the 10 subjects, 1 subject was found to have increased platelet aggregation (blood passing time $>70$ s) after 1 week of $\mathrm{SCH}$. The data from this particular subject was excluded from the study.

\section{Effect of SCH Intake}

Following continuous intake of Schisandra for 2 weeks the fasting blood passage time was reduced by $9.0 \%$ and $9.7 \%$ at week 1 and week 2, respectively. The baseline blood passage time prior to intake of SCH tablets was $53.5 \pm 3.5 \mathrm{sec}$ as compared to $47.2 \pm 3.2 \mathrm{sec}$ and $47.2 \pm 1.7 \mathrm{sec}$ at Week 1 and

ISSN 0973-2063

Bioinformation 2(6): 249-252 (2008)
Week 2. Although this change was not statistically significant, there was a sustained decreasing trend in blood passage time during 2 weeks of Schisandra administration (Figure 1).

\section{Safety Parameters}

Haematology, blood biochemistry, and urine tests did not show any abnormal change throughout the study period of $\mathrm{SCH}$ administration. The liver function indicators and plasma lipids measured before and after $\mathrm{SCH}$ administration is shown in Table 1 (see supplementary material). The 
baseline activities of these enzymes, ALT, AST, ALP and $\gamma$ GT were similar to that at week 2 . The baseline pre-study plasma levels of total cholesterol, HDL- cholesterol, LDLcholesterol and triglycerides were marginally higher than the reference range and 2 weeks of SCH treatment did not cause any significant change in lipid parameters.

\section{Discussion:}

In human subjects, the fasting blood passing time shows a sustained decreasing trend over the two-week trial period, suggesting that $\mathrm{SCH}$ may have beneficial effect in promoting blood fluidity. The blood passage time following $\mathrm{SCH}$ intake were similar at week 1 and week 2 when compared to baseline (week 0), suggesting that a limit of action was reached within that time frame. As shown in figure 1, the blood passage time stabilized at $47 \mathrm{sec}$ and did not fall below the pre-determined 'normal' blood passage time $(\leq 44 \mathrm{sec})$, it appears that $\mathrm{SCH}$ has a sustained beneficial effect on blood fluidity. This ceiling of activity in reducing blood viscosity and no undesirable change in haematology, liver function indicators, lipid profiles and urine tests also infer safety for the consumption of sesaminschisandra extract combination.

Various chinese medicinal herbs claim to increase blood circulation and combat "blood stasis" in humans [15] (Shen and Cui, 1998). Scientific research has substantiated the ability of several herbs to reduce blood viscosity, and in the case of the popular Ginko biloba, platelet aggregation [15] Herbal combinations with demonstrated clinical effects in human subjects include 'Pushen' [16] and 'Dahuang Zhechong Wan' [17]. Another well-known natural remedy is garlic (Allium sativum), which exhibits blood viscosity reducing quality and is associated with a reduction in cholesterol levels, platelet aggregation and overall reduction in cardiovascular disease risk [18]. Kiesewetter and colleagues [19] discussed that improved blood fluidity attributed to garlic can increase capillary perfusion which can have a cleansing effect, contributing to 'purification' of the microcirculation.

In contrast to Gingko biloba and garlic, where a reduction in platelet aggregation and/or plasma lipids is the proposed mechanism for reducing blood viscosity, sesamin in combination with schisandra extract might work by an indirect route of enhancing liver metabolic functions. A recent DNA microarray study indicated that sesamin regulates the metabolism of lipids, xenobiotics, and alcohol at the mRNA level [20]. Moreover, several studies supported the liver protective efficacy of sesamin and schisandra extract [10, 12 - 13]. In support of our hypothesis, Xie and colleagues [2] showed that a combination of 4 herbs including 'Dang gui' (Angelica Sinensis) and 'Dan Shen'
(Salviae Miltiorrhizae) could improve blood circulation whilst reducing oxidative damage in mice with chronic liver damage. Together with the current data, this presents a link between liver protection and blood circulation.

Considering this is the first pilot clinical study on the combined efficacy and safety of sesamin and schisandra extract, and majority of data is derived from animal studies, it warrants further investigations to confirm the role of sesamin and schisandra extract in improving blood fluidity and flow. In conclusion, this study indicates that $\mathrm{SCH}$ can enhance blood fluidity without causing undesirable side effect.

\section{References:}

[01] R. Q. Qian, et al., Zhongguo Zhong Xi Yi Jie He Za Zhi, 21: 364 (2001) [PMID: 12577423]

[02] F. Xie, et al., J. Tradit. Chin. Med., 21: 225 (2001) [PMID: 11789334]

[03] A. R. Saniabadi, et al., Thromb. Haemost., 77: 996 (1997) [PMID: 9184417]

[04] D. Eterovic, et al., Clin. Hemorheol., 15: 841 (1995)

[05] J. F. Brun, et al., Clin Hemorheol., 13: 201 (1993)

[06] K.Wang, et al., Zhonghua Shi Yan He Lin Chuang Bing Du Xue Za Zhi, 17: 280 (2003) [PMID: 15340578]

[07] T. Kurihara, et al., Clin. Hemorheol. Microcirc., 24: 217 (2001) [PMID: 11564910]

[08] Q. M. Wang \& J. Z. Shi. Chin. Med. J. (Engl)., 103: 939 (1990) [PMID: 2125916]

[09] H. Nobuaki, et al., J. Lipid Res., 32: 629 (1991)

[10] M. Sugano \& K.Akimoto, J. Chi. Nutr. Soc., 18: 1 (1993)

[11] M. Zhu, et al., Planta. Med., 66: 521 (2000) [PMID: 10985077]

[12] P. Y. Pan, et al., Planta. Med., 68: 217 (2002)

[13] K. C. Huang, The Pharmacology of Chinese Herbs. $2^{\text {nd }}$ Ed. CRC Press LLC, USA (1998)

[14] Y. Kikuchi, Microvasc. Res., 50: 288 (1995) [PMID: 8538506]

[15] L. Shen, \& Y. Cui, Zhongguo Zhong Yao Za Zhi, 23: 622 (1998) [PMID: 11599364]

[16] Z. L. Liu, et al., Zhongguo Zhong Xi Yi Jie He Za Zhi, 24: 227 (2001)

[17] Y. M. Huang \& S. F. Shen., Zhong Xi Yi Jie He Za Zhi, 9: 589 (1989) [PMID: 2605735]

[18] G. Siegel, et al., Wien Med Wochenschr, 149: 217 (1999) [PMID: 10483684]

[19] H. Kiesewetter, et al., Br. J. Clin. Pract., 69: 24 (1990) [PMID: 2128029]

[20] N. Tsuruoka, et al., Biosci Biotechnol Biochem., 69: 179 (2005)[PMID:15665483]

Edited by P. Kangueane

Citation: Tsi \& Tan, Bioinformation 2(6): 249-252 (2008) License statement: This is an open-access article, which permits unrestricted use, distribution, and reproduction in any medium, for non-commercial purposes, provided the original author and source are credited. 


\section{Supplementary material}

\begin{tabular}{lll}
\hline \multicolumn{1}{c}{ Blood Chemistry } & \multicolumn{1}{c}{ Baseline* } & \multicolumn{1}{c}{ Week 2 } \\
\hline Liver function indicator & $\mathbf{U} / \mathbf{L}$ & $\mathbf{U} / \mathbf{L}$ \\
Aminotransferase (ALT) & $44.8 \pm 13.0$ & $45.2 \pm 13.5$ \\
Aspartate aminotransferase (AST) & $31.3 \pm 4.4$ & $31.1 \pm 6.0$ \\
Alkaline Phosphatase (ALP) & $248.4 \pm 3.6$ & $252.8 \pm 15.6$ \\
Gamma-glutamyltransferase $\gamma$-GT & $65.7 \pm 3.8$ & $63.4 \pm 13.6$ \\
Plasma lipid & $\mathbf{~ m g / d L ~}$ & $\mathbf{~ m g / d L}$ \\
Total cholesterol & $220.1 \pm 8.27$ & $219.0 \pm 7.31$ \\
HDL-Cholesterol & $52.4 \pm 3.69$ & $51.0 \pm 2.77$ \\
LDL-Cholesterol & $139.7 \pm 5.42$ & $139.2 \pm 5.11$ \\
Triglycerides & $135.4 \pm 24.14$ & $138.1 \pm 13.63$ \\
\hline
\end{tabular}

Table 1: Blood Chemistry showing results of liver function test and plasma lipid levels in subjects before and after administration with $\mathrm{SCH}$. SCH supplementation for 2 weeks did not cause any undesirable change in liver function indicators and plasma lipid profiles of the subjects. Values represent Mean \pm SEM. ${ }^{*}$ Measurements were taken 2 weeks prior to commencement of SCH intake. 